\title{
Shock Structure of the High-Velocity Water Masers in W49 N
}

\author{
T. Liljeström \\ Metsähovi Radio Research Station, Helsinki University of Technology, Finland
}

\begin{abstract}
Magnetic field parameters and other characteristics of the postshocked gas in W49 $\mathrm{N}$ are derived from a linewidth comparison between the multi-epoch VLBI data of Gwinn and contemporaneous long-term single-dish observations. The observations support predictions of dissociative shock models.
\end{abstract}

Dissociative shocks caused by energetic protostellar jets and outflows running into dense magnetized material successfully explain the high-velocity water maser emission (Hollenbach \& McKee 1989; Elitzur, Hollenbach, \& McKee 1989; Hollenbach, Elitzur, \& McKee 1993). These comprehensive shock models have been somewhat difficult to probe observationally due to many free parameters. However, combining the VLBI data of W49 N (Gwinn, Moran, \& Reid 1992; Gwinn 1994a,b,c) and the Metsähovi data base of 146 maser outbursts (Liljeström et al. 1989), obtained during Gwinn's multi-epoch VLBI observations, all free parameters in the shock model are fixed allowing a straightforward determination of the shock structure of the high-velocity water masers in $\mathrm{W} 49 \mathrm{~N}$.

A critical parameter determining the shock structure is the magnetic pressure, which dominates in the masing region and which is set by the ram pressure of the shock (Hollenbach, Elitzur, \& McKee 1993). Consequently the strength of the magnetic field in the masing region is independent of the preshock magnetic field. With a preshock hydrogen nuclei density of $410^{6} \mathrm{~cm}^{-3}$ (see Serabyn, Güsten, \& Schulz 1993) and a typical shock velocity of $100 \mathrm{~km} \mathrm{~s}^{-1}$, equivalent to the median space velocity of masers moving at space velocities greater than $45 \mathrm{~km} \mathrm{~s}^{-1}$ (see Table 4 of Gwinn, Moran, \& Reid 1992), the dissociative shock model (Hollenbach \& McKee 1989; Elitzur, Hollenbach, \& McKee 1989; Hollenbach, Elitzur, \& McKee 1993) predicts a total magnetic field strength of typically $126 \mathrm{mG}$ in the masing region. This is in good agreement with the measured maximum field value along the line of sight toward W49 N (90 mG for the $\mathbf{F}=6-5$ hyperfine transition, see Fiebig \& Güsten 1989), from which a total magnetic field around $120 \mathrm{mG}$ results for an inclination of $40^{\circ}$ obtained by Gwinn, Moran, \& Reid (1992).

An estimate of the preshock magnetic field (perpendicular to the shock velocity) is derived from a linewidth comparison of the VLBI and single-dish data (which noteworthy have the same velocity resolution). For high-velocity water maser features the typical Doppler linewidth (FWHM) in the VLBI data of Gwinn (1994a,b) is $0.7 \mathrm{kms}^{-1}$ (C. Gwinn, private communication), whereas in the single-dish data the distribution of the linewidths at outburst maximum peaks at $1.1 \mathrm{kms}^{-1}$ for the high-velocity masers. An obvious explanation for this difference is that the linewidth derived from the VLBI data does not reveal the line broadening caused by the magnetic pressure. If one includes into the linewidth consideration both the broadening produced by the scattering in the maser halo of W49 N (FWHM about $0.31 \mathrm{kms}^{-1}$, see Gwinn 1994c) and the effect of the magnetic pressure, then the single-dish linewidth requires $0.79 \mathrm{kms}^{-1}$ (FWHM) for the magnetic broadening. Taking advantage of the often assumed equipartition relation between the different energy modes (e.g., Myers \& Goodman 1988), we estimate that the non-fluctuating part of the linewidth (caused 
by the magnetic pressure) roughly equals the fluctuating part of the magnetic energy (in form of Alfven waves). This yields a non-thermal velocity dispersion of $0.34 \mathrm{kms}^{-1}$, an Alfven velocity of $0.59 \mathrm{kms}^{-1}$, a perpendicular Alfven Mach number of 170 , and a preshock magnetic field of $0.64 \mathrm{mG}$ (see Hollenbach \& McKee 1979, 1989). With these observationally fixed magnetic field quantities it is straightforward to derive the physical and chemical parameters of the postshocked gas.

In the list below the value outside (inside) the parenthesis stands for masers outside (inside) the ambient cloud velocity range $\left(0-16 \mathrm{kms}^{-1}\right)$. Inside the ambient cloud velocity range the distribution of the single-dish linewidths at outburst maximum peaks at $1.3 \mathrm{kms}^{-1}$ whereas the VLBI linewidths (FWHM) peak at $0.5 \mathrm{kms}^{-1}$ (Gwinn 1994a and private communication). This suggests that inside the ambient cloud velocity range the non-thermal velocity dispersion is $0.5 \mathrm{kms}^{-1}$ and the Alfven velocity $0.87 \mathrm{kms}^{-1}$. Assuming that the rate coefficient for $\mathrm{H}_{2}$ formation on grains is the same for all high-velocity masers, we find that a shock velocity of $50 \mathrm{kms}^{-1}$ (representative for the lower end of the dissociative shock velocities) requires a preshock density of $1.710^{7} \mathrm{~cm}^{-3}$. This yields a preshock magnetic field of $1.9 \mathrm{mG}$; in the masing region the field is $130 \mathrm{mG}$ (i.e., the same as for masers outside the ambient cloud). With these parameters the other postshock quantities are as follows:

Hydrogen nuclei density in the masing region: $9.610^{8} \mathrm{~cm}^{-3}\left(1.410^{9} \mathrm{~cm}^{-3}\right)$; compression factor $\mathrm{n} / \mathrm{n}_{0}: 240(82)$; rate coefficient for $\mathrm{H}_{2}$ formation on grains: $0.2610^{-17} \mathrm{~cm}^{-3} \mathrm{~s}^{-1} ; \mathrm{H}_{2}$ formation time behind the dissociative shock (i.e., the time needed to convert all $\mathrm{H}$ to $\mathrm{H}_{2}: 13 \mathrm{yr}(6 \mathrm{yr})$; temperature plateau of the masing region: $320 \mathrm{~K}(360 \mathrm{~K})$; hydrogen column in the temperature plateau: $1.610^{22} \mathrm{~cm}^{-2}\left(2.410^{22} \mathrm{~cm}^{-2}\right)$; thickness of the temperature plateau: $1.14 \mathrm{AU}$ (1.12 AU), which is in excellent agreement with Gwinn's result (1994a) obtained from a two-point correlation function of the maser features; water abundance in the masing region: $1.210^{-4}\left(6.610^{-5}\right)$; water density in the masing region: $1.110^{5} \mathrm{~cm}^{-3}\left(9.210^{4} \mathrm{~cm}^{-3}\right)$; and water column density: $1.910^{18} \mathrm{~cm}^{-2}$ $\left(1.610^{18} \mathrm{~cm}^{-2}\right)$.

\section{References}

Elitzur, M., Hollenbach, D. J., \& McKee, C. F. 1989. ApJ, 346, 983-990.

Fiebig, D., \& Güsten, R. 1989. A6 A, 214, 333-338.

Gwinn, C. R. 1994a. ApJ, 429, 241-252.

Gwinn, C. R. 1994b. ApJ, 429, 253-267.

Gwinn, C. R. 1994c. ApJ, 431, L123-126.

Gwinn, C. R., Moran, J. M., \& Reid, M. J. 1992. ApJ, 393, 149-164.

Hollenbach, D. J., \& McKee, C. F. 1979. ApJS, 41, 555-592.

Hollenbach, D. J., \& McKee, C. F. 1989. ApJ, 342, 306-336.

Hollenbach, D. J., Elitzur, M., \& McKee, C. F. 1993. In Astrophysical Masers, eds. A. Clegg \& G. Nedoluha (Berlin: Springer), 159-163.

Liljeström, T., et al. 1989. A\&\&AS, 79, 19-39.

Myers, P. C., \& Goodman, A. A. 1988. ApJ, 329, 392-405.

Serabyn, E., Güsten, R., \& Schulz, A. 1993. ApJ, 413, 571-581. 